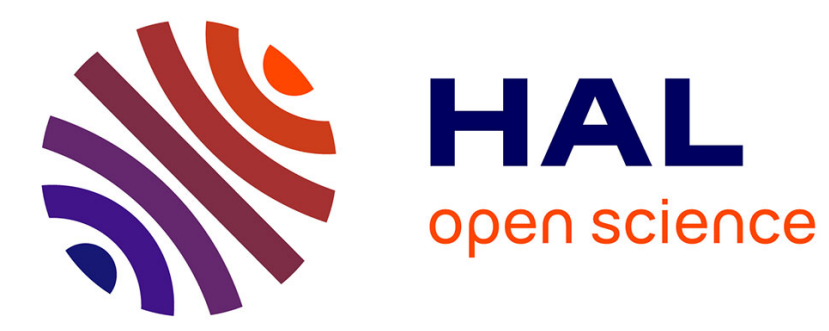

\title{
Investigation of the Torsional Effects on the Lateral Buckling of a Pipe-Like Beam Resting on the Ground under Axial Compression
}

Philippe Le Grognec, Alain Nême, Jie Cai

\section{- To cite this version:}

Philippe Le Grognec, Alain Nême, Jie Cai. Investigation of the Torsional Effects on the Lateral Buckling of a Pipe-Like Beam Resting on the Ground under Axial Compression. International Journal of Structural Stability and Dynamics, 2020, 20 (09), pp.2050110. 10.1142/S0219455420501102 . hal02995578

HAL Id: hal-02995578

https://hal-ensta-bretagne.archives-ouvertes.fr/hal-02995578

Submitted on 11 May 2021

HAL is a multi-disciplinary open access archive for the deposit and dissemination of scientific research documents, whether they are published or not. The documents may come from teaching and research institutions in France or abroad, or from public or private research centers.
L'archive ouverte pluridisciplinaire HAL, est destinée au dépôt et à la diffusion de documents scientifiques de niveau recherche, publiés ou non, émanant des établissements d'enseignement et de recherche français ou étrangers, des laboratoires publics ou privés. 


\title{
Investigation of the torsional effects on the lateral buckling of a pipe-like beam resting on the ground under axial compression
}

\author{
Philippe Le Grognec ${ }^{\mathrm{a}, *}$, Alain Nême ${ }^{\mathrm{a}}, \mathrm{Jie}_{\mathrm{Cai}}^{\mathrm{a}}$ \\ ${ }^{a}$ ENSTA Bretagne, UMR CNRS 6027, IRDL, F-29200 Brest, France
}

\begin{abstract}
This paper deals with the lateral buckling behavior of an axially compressed beam interacting with the ground on which it is resting. Such a simple model is supposed to reproduce the same trends as observed during the lateral buckling of offshore pipelines on the seabed. In such practical analyses, the pipe-soil interaction relies the ground to the neutral axis of the pipeline. It is shown that, although such a constraint significantly affects the buckling behavior of the pipeline, it can not reflect the torsional component of the buckling modes. However, this component is encountered in practice and may further modify the critical loads. Therefore, in the present preliminary study, the interaction between the beam in hand and the surrouding ground is modeled by a connection (a continuous distribution of lateral springs) related to the bottom line of the beam. In this way, the real contact between the soil and the bottom line of a pipe is mimicked, allowing for both flexural and torsional deformations in the buckling response. The problem is investigated analytically, using an Euler-Bernoulli beam model with an isotropic linear elastic constitutive law and also an elastic interaction law. Original analytical solutions are derived and compared to numerical results obtained through finite element computations. In comparison with classical solutions (with the connection related to the neutral axis), new types of buckling modes may appear when considering torsional effects, depending on the boundary conditions, with generally much lower critical loads. These first results are certainly representative of some features of the global/localized lateral buckling of offshore pipelines, indicating that torsional effects should also be taken into account in such more comprehensive analyses.
\end{abstract}

Keywords: Lateral buckling, Torsional effects, Analytical solutions, Numerical simulation, Submarine pipelines

\section{Nomenclature}

П $\quad$ First Kirchhoff stress tensor

$\boldsymbol{\Sigma} \quad$ Second Kirchhoff stress tensor

\footnotetext{
* Corresponding author.

Email address: philippe.le_grognec@ensta-bretagne.fr (Philippe Le Grognec)
} 
$\Lambda_{c} \quad$ Critical stress

$\omega_{x} \quad$ Cross-section torsional rotation of the beam

C Fourth-order material tangent elastic reduced tensor

D Fourth-order material tangent elastic tensor

E Green strain tensor

F Deformation gradient

K Fourth-order nominal tangent elastic tensor

U Displacement field

X Bifurcation mode

A Cross-section area of the pipe

E Young's modulus

$F_{z} \quad$ Lateral force

G Shear modulus

$g \quad$ Transverse slip along the interface

I Flexural second moment of area of the pipe

$J \quad$ Torsional second moment of area of the pipe

$k \quad$ Stiffness density by unit length of the continuous spring distribution

$K_{d} \quad$ Discrete lateral stiffness

$M_{x} \quad$ Torsional moment

$P_{c} \quad$ Critical force

$R_{e}, R$ External radius of the pipe

$R_{i} \quad$ Internal radius of the pipe

$u_{x} \quad$ Axial displacement of the centroid axis of the beam

$u_{z} \quad$ Transverse displacement of the centroid axis of the beam 


\section{Introduction}

Owing to the growing development and utilization of offshore oil and gas resources, the mechanical design of offshore pipelines becomes increasingly important. The actual loading conditions coming into play in the mechanical behavior of submarine pipelines are complex and variable during the entire life cycle of

5 such structures. The typical loads exerted on pipelines include internal pressure, external pressure, bending moments, axial and shear forces (among which frictional forces), and even torsional forces [1]. As a result of all these conditions, lateral buckling turns out to be one of the main failure modes of such long pipelines resting on the seabed, and it must be thus particularly investigated for dimensioning purposes.

In practice, the buckling of a pipeline is naturally due to longitudinal compressive stresses that may arise from various solicitations such as internal and external pressures, but also temperature gradients, among others. Indeed, in deep sea environment, high temperatures (HT) and high pressures (HP) are usually deployed in order to maintain the regular operation of hydrocarbon contents. The temperature of oil and gas pipelines may be up to $100^{\circ} \mathrm{C}$ above the water environment, while the internal pressure may be higher than $10 M P a$ [2, 3. Under such loading conditions, pipelines are prone to buckle laterally in a global or more often local way [4, 5] (the possible local failure of pipelines due to extra bending after overall buckling will not be discussed in this paper, see [6, 7] for more details on this topic). The considerable length of submarine pipelines, relatively to their diameter, makes the use of beam models perfectly natural for modeling purposes. In this framework, the concept of effective axial force has been therefore introduced so as to integrate in a simplified way the combined effects of the thermal expansion and the internal/external pressures, without involving any complex integral on the double-curved surface of the pipe due to pressures, what allows one to deal easily with global buckling, for instance. For detailed derivations of this controversial but widely used effective axial force, please refer to researchers such as Palmer and Baldry [8], Fyrileiv and Collberg [9] and Vedeld et al. [10, or to engineering standards (DNV).

Despite the fact that a pipeline behaves like a slender beam under axial compression, the classical Euler 25 buckling loads are not suitable, mainly because of the pipe-soil interaction that plays a major role in the lateral buckling response. When a pipeline is resting on the seabed, the pipe-soil interaction depends on many parameters and hence several models have been deployed to date. In traditional pipeline investigation, typical pipe-seabed models such as the bi-linear [11, 12] and tri-linear [13, 14, 15] models have been developed and widely used. The soil berm effect has also been included in some research such as [16]. Wang and van der Heijden [5] made use of a more realistic non-linear pipe-soil interaction model that accounts for the effect of lateral breakout resistance. The use of these pipe-seabed models depends on the type of seabed, which may consist in soft clay [17, 13, 18, stiff clay [16] or sand [19]. In practice, the pipe is supposed to penetrate into the seabed with a finite depth, giving rise to a finite contact area between the pipe bottom and the seabed. Wang et al. 20] and Zeng and Duan 21] explicitly investigated this specific configuration, 
considering the case of a soft seabed. However, for simplicity purposes, the actual uneven seabed is often assumed to be flat, without disturbances, also excluding any penetration effect (see Hobbs 22, 23, Taylor and Gan [2, Ju and Kyriakides [24, Taylor and Tran [25, Karampour et al. [3] and Hong et al. [26, for instance). The conventional design practice for the pipe-soil interaction assumes thus classically a uniform frictional behavior in the horizontal direction (proportional to the pipe effective weight underwater, in the simplest case) [27, 23]. With these simplifications, spring elements shall be used to represent the resistance force in the pipe-soil interaction, assuming the seabed to be rigid [28, 11, 29, 19].

To the best of the authors' knowledge, there are no studies in the literature dealing with the particular role of torsion in the lateral buckling analysis of submarine pipelines (the spring elements are invariably connected to the neutral axis of the pipe, and thus the lateral force is applied thereto, so that no torsion is supposed to occur). In practice, torsional displacements are assumed to be small and torsion is thus ignored for pipes with large slenderness in most current research [30, 31. However, with the assumption of a rigid seabed and ignoring the pipe embedment, the lateral "frictional" force may rather be applied on the bottom line of the pipe, in real contact with the seabed, instead of the neutral axis. Under this circumstance, the torsion effects may have a large influence on the lateral buckling of pipelines and cannot be neglected.

So far, there have been a large number of papers dealing with the lateral buckling of pipelines, aiming at predicting critical loads and buckling modes with analytical or numerical models (see 23, 2, 32, among others). The use of numerical software is clearly interesting as it allows one to include many physical phenomena, without too many simplifications, and to deal with the post-buckling response of pipes, taking into account both geometric and material non-linearities. Meanwhile, several analytical models have been recently proposed in the literature for this problem [26, 31, with more or less severe assumptions concerning the geometry, constitutive law, loading/boundary conditions and interactions, which bring a more straightforward understanding of the lateral buckling phenomenon with more efficient general (mostly closed-form) solutions.

Therefore, in this paper, a bifurcation analysis of a perfect hollow circular cylindrical tube under axial compression is carried out, accounting for torsional effects. The lateral (global) buckling is investigated, considering an elastic connection between the bottom line of the tube and the ground on which it is laid down. This specific constraint gives rise to original analytical, possibly closed-form, solutions depending on the boundary conditions. The paper is organized as follows. In Section 2, use is made of linearized bifurcation theory so as to solve the buckling problem. This approach is a general and efficient way to cope with the buckling of structures subjected to uniform pre-critical stress states. Among others, it was successfully applied to the cases of compressed homogeneous beams [33, sandwich beam-columns [34, 35] and two-layer beams with partial interaction (with similar connections) [36, 37, in earlier studies. The tube is represented by an Euler-Bernoulli beam model with an isotropic linear elastic material, and the ground-structure interaction is described using a uniform distribution of elastic lateral springs along the 
beam length, connected to the bottom line of the tube in contact with the soil. The final set of differential equations is analytically solved as far as the boundary conditions make it possible. In Section 3 numerical finite element computations are performed for purposes of comparison with the previous analytical solutions. The new expressions are also confronted to the solutions obtained without considering torsional effects in the buckling response. Two sets of boundary conditions are retained and the influence of the stiffness of the lateral connectors is particularly examined. The results show the importance of torsional effects in such a buckling analysis.

\section{Analytical study of the lateral buckling of an axially compressed beam}

\subsection{Problem definition}

Let us consider a straight beam, with a hollow circular constant cross-section, resting on the ground. The bottom line of the cylindrical tube (in contact with the ground) is connected by a continuous distribution of lateral springs to the line on the ground representing the initial position of the beam (it means that the return springs are not stressed in the reference configuration, when the two lines coincide), see Figure 1 . The geometry of the beam is defined by its length $L$ (with endpoints located at $x=0$ and $x=L$ ) and its internal and external radii $R_{i}$ and $R_{e}$, respectively. Later, use will be made of the cross-section area $A$, and the flexural and torsional second moments of area $I$ and $J$, respectively, which can be expressed in an exact form as follows:

$$
\begin{aligned}
& A=\pi\left(R_{e}^{2}-R_{i}^{2}\right) \\
& I=\frac{\pi\left(R_{e}^{4}-R_{i}^{4}\right)}{4} \\
& J=\frac{\pi\left(R_{e}^{4}-R_{i}^{4}\right)}{2}
\end{aligned}
$$

The material of the beam is assumed to be homogeneous, isotropic and linear elastic, and the corresponding 3D constitutive law is defined by the fourth-order elasticity tensor $\mathbf{D}$ whose components in an orthonormal basis are $D_{i j k l}=\lambda \delta_{i j} \delta_{k l}+\mu\left(\delta_{i k} \delta_{j l}+\delta_{i l} \delta_{k j}\right)$, where $\delta_{i j}$ is the Kronecker symbol, and $\lambda$ and $\mu$ are the Lamé constants. Use will be made of the Young's modulus $E$ (which is related to $\lambda$ by the standard relation $\lambda=\frac{E \nu}{(1+\nu)(1-2 \nu)}$ also involving the Poisson's ratio $\nu$ ) and the shear modulus $G=\mu$ (related to $E$ and $\nu$ by $\left.G=\frac{E}{2(1+\nu)}\right)$. The lateral stiffness density by unit length of the continuous spring distribution, denoted by $k$, is supposed to be uniform and constant. There is no need here for a non-linear interaction law, since only the initial stiffness is involved in the present linearized buckling analysis.

The beam thus defined is subjected to an axial compressive force $P$ which leads to lateral buckling. The critical loads and the bifurcation modes are subsequently derived from a 3D framework, using a total Lagrangian formulation. 


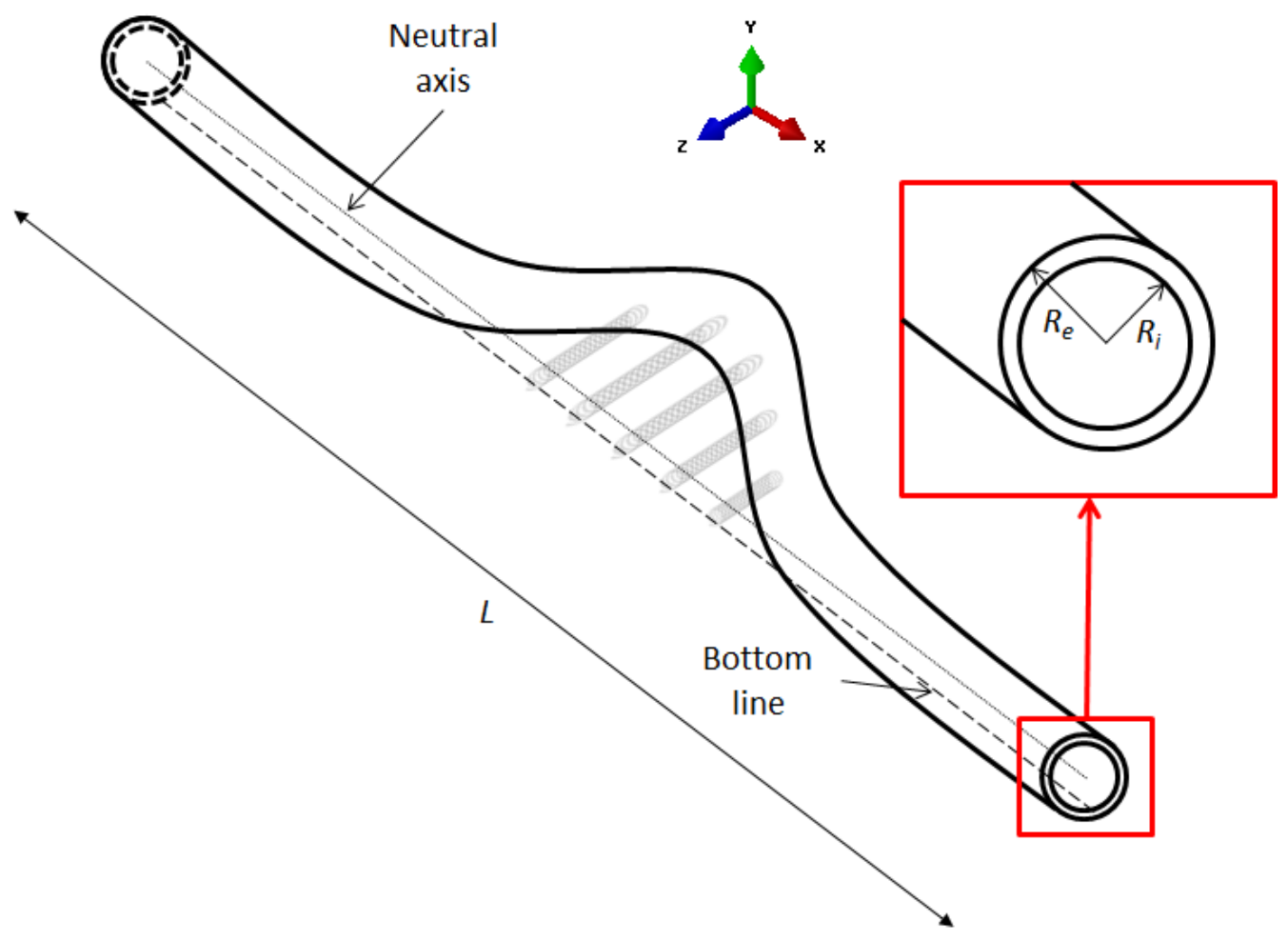

Figure 1: Cylindrical tube with lateral connectors under axial compression

\subsection{Theoretical formulation}

The critical loading $\Lambda_{c}$ and the bifurcation mode $\mathbf{X}$ of a 3D body, occupying $\Omega$ in the reference configuration, can be classically obtained by solving the following bifurcation equation [38]:

$$
\forall \delta \mathbf{U} \quad(\mathrm{KA}), \quad \int_{\Omega} \nabla^{T} \delta \mathbf{U}: \mathbf{K}\left(\Lambda_{c}\right): \nabla \mathbf{X} d \Omega=0
$$

where $\delta \mathbf{U}$ can be considered as a kinematically admissible test function or, more physically, as the virtual variation of the unknown displacement field $\mathbf{U}$.

The fourth-order nominal tangent elastic tensor $\mathbf{K}$ can be written as follows:

$$
\mathbf{K}=\frac{\partial \boldsymbol{\Pi}}{\partial \mathbf{F}}=\mathbf{F} \cdot \frac{\partial \boldsymbol{\Sigma}}{\partial \mathbf{E}} \cdot \mathbf{F}^{T}+(\mathbb{I} \cdot \boldsymbol{\Sigma})^{T}=\mathbf{F} \cdot \mathbf{D} \cdot \mathbf{F}^{T}+(\mathbb{I} \cdot \boldsymbol{\Sigma})^{T}
$$

In the above equation, $\mathbf{E}$ denotes the Green strain tensor and $\boldsymbol{\Sigma}$ the second Kirchhoff stress tensor (symmetric); $\mathbf{F}$ is the deformation gradient and $\mathbf{\Pi}=\mathbf{F} . \boldsymbol{\Sigma}$ the first Kirchhoff stress tensor (non-symmetric); I represents the fourth-order unit tensor $\left(I_{i j k l}=\delta_{i l} \delta_{k j}\right)$ and the superscript ${ }^{T}$ the transposition of a secondorder tensor and the major transposition of a fourth-order tensor $\left(\left(\mathbf{A}^{T}\right)_{i j k l}=A_{k l i j}\right)$, respectively. Use is also made of the fourth-order material tangent elastic tensor $\mathbf{D}$ which has been already introduced.

More explicit expressions of the above tensors shall now be derived by exploiting the uniaxial stress state 
compressive stress $\Pi_{x x}=-\Lambda<0$, so that the first Kirchhoff stress tensor $\Pi$ is expressed in the orthonormal basis $\left(\mathbf{e}_{x}, \mathbf{e}_{y}, \mathbf{e}_{z}\right)$ as:

$$
\boldsymbol{\Pi}=-\Lambda \mathbf{e}_{x} \otimes \mathbf{e}_{x}=\left[\begin{array}{ccc}
-\Lambda & 0 & 0 \\
0 & 0 & 0 \\
0 & 0 & 0
\end{array}\right] \quad(\Lambda>0)
$$

Let us make the assumption that the pre-critical deformations are small, which is usually satisfied in practice:

$$
\|\nabla \mathbf{U}\| \ll 1
$$

Thus, the stress tensor $\boldsymbol{\Sigma}$ writes:

$$
\Sigma=\mathbf{F}^{-1} . \Pi \approx \Pi
$$

The nominal tangent elastic tensor in Equation (3) becomes:

$$
\mathbf{K} \approx \frac{\partial \boldsymbol{\Sigma}}{\partial \mathbf{E}}+(\mathbb{I} \cdot \boldsymbol{\Sigma})^{T}=\mathbf{D}-\Lambda \mathbf{e}_{i} \otimes \mathbf{e}_{x} \otimes \mathbf{e}_{x} \otimes \mathbf{e}_{i}
$$

which is independent of the spatial coordinates (the implicit summation convention on repeated indices is used with $i=x, y, z)$.

Furthermore, when dealing with 1D models like beams, ad hoc assumptions are usually added in order to enforce some specific stress state in the body. Namely, the transverse normal material stresses are assumed to be zero: $\Sigma_{y y}=\Sigma_{z z}=0$. Taking into account these assumptions leads one to replace tensor $\mathbf{D}$ with the reduced tensor $\mathbf{C}$ defined as:

$$
\begin{array}{r}
C_{i j k l}=D_{i j k l}+\frac{D_{i j y y}\left(D_{y y z z} D_{z z k l}-D_{z z z z} D_{y y k l}\right)+D_{i j z z}\left(D_{z z y y} D_{y y k l}-D_{y y y y} D_{z z k l}\right)}{D_{y y y y} D_{z z z z}-D_{y y z z} D_{z z y y}} \\
(i, j) \neq(y, y),(z, z) \quad(k, l) \neq(y, y),(z, z)
\end{array}
$$

It can be readily checked that tensor $\mathbf{C}$ has the major and both minor symmetries. Below, only the following reduced moduli (and their equivalents obtained by major or minor symmetries) will be needed:

$$
C_{x x x x}=E \quad C_{x y x y}=C_{x z x z}=C_{y z y z}=\mu=G
$$

Eventually, the bifurcation equation (2) of a beam writes in the uniaxial stress case:

$$
\forall \delta \mathbf{U} \quad(\mathrm{KA}), \quad \int_{\Omega} \nabla^{T} \delta \mathbf{U}:\left(\mathbf{C}-\Lambda_{c} \mathbf{e}_{i} \otimes \mathbf{e}_{x} \otimes \mathbf{e}_{x} \otimes \mathbf{e}_{i}\right): \nabla \mathbf{X} d \Omega=0
$$

As far as a connected beam is concerned, one has to consider the previous integral (Equation (100) in the global bifurcation equation together with a special term for the contribution of the connectors, which will be formulated later.

Let us now consider the bending-twisting problem of the considered beam in the $x z$-plane (uplift is not introduced in the model, see Figure 1). Owing to the high slenderness ratio of the designed pipelines in practice, transverse shear effects may be negligible, so that the Euler-Bernoulli theory is retained for the 
beam in hand. The Euler-Bernoulli kinematics is defined here by two scalar displacement fields $u_{x}(x)$ and $u_{z}(x)$, respectively the axial and transverse displacements of the centroid axis of the beam, and the crosssection torsional rotation $\omega_{x}(x)$ about the same axis (the flexural rotation about the $y$-axis is directly related to the transverse displacement field since the cross-sections are supposed to remain orthogonal to the centroid axis of the beam after deformation). When the beam buckles from the straight position (the fundamental solution) to a bent-twisted shape, the expressions for the bifurcation mode $\mathbf{X}$ and the displacement variation $\delta \mathbf{U}$ are both chosen according to the following kinematics:

$$
\mathbf{X}=\left|\begin{array}{l}
u_{x}-z u_{z, x} \\
-z \omega_{x} \\
u_{z}+y \omega_{x}
\end{array} \quad \delta \mathbf{U}=\right| \begin{aligned}
& \delta u_{x}-z \delta u_{z}, x \\
& -z \delta \omega_{x} \\
& \delta u_{z}+y \delta \omega_{x}
\end{aligned}
$$

The bifurcation mode gradient then writes:

$$
\nabla \mathbf{X}=\left[\begin{array}{ccc}
u_{x}, x-z u_{z}, x x & 0 & -u_{z, x} \\
-z \omega_{x}, x & 0 & -\omega_{x} \\
u_{z, x}+y \omega_{x, x} & \omega_{x} & 0
\end{array}\right]
$$

and the gradient of the displacement variation takes a similar form.

The contribution of the connectors in the bifurcation equation involves the (modal) transverse slip $g$ along the interface, which can be expressed as follows:

$$
g=u_{z}-R_{e} \omega_{x}
$$

where $R_{e}$ will be further denoted by $R$ for brevity purposes.

The global bifurcation equation then writes:

$$
\forall \delta \mathbf{U} \quad(\mathrm{KA}), \quad \int_{\Omega} \nabla^{T} \delta \mathbf{U}:\left(\mathbf{C}-\Lambda_{c} \mathbf{e}_{i} \otimes \mathbf{e}_{x} \otimes \mathbf{e}_{x} \otimes \mathbf{e}_{i}\right): \nabla \mathbf{X} d \Omega+\int_{0}^{L} \delta g k g d x=0
$$

that is to say:

$$
\begin{aligned}
& \forall \delta u_{x}, \delta u_{z}, \delta \omega_{x} \quad(\mathrm{KA}), \quad \int_{\Omega}\left[E\left(u_{x},{ }_{x}-z u_{z, x x}\right)\left(\delta u_{x},,_{x}-z \delta u_{z},{ }_{x x}\right)+y^{2} G \omega_{x},{ }_{x} \delta \omega_{x},{ }_{x}+z^{2} G \omega_{x},_{x} \delta \omega_{x}, x\right. \\
& -\frac{P_{c}}{A}\left(u_{x},,_{x}-z u_{z, x x}\right)\left(\delta u_{x}, x-z \delta u_{z},{ }_{x x}\right)-\frac{P_{c}}{A} z^{2} \omega_{x, x} \delta \omega_{x}, x \\
& \left.-\frac{P_{c}}{A}\left(u_{z, x}+y \omega_{x}, x\right)\left(\delta u_{z, x}+y \delta \omega_{x}, x\right)\right] d \Omega+\int_{0}^{L} k\left(u_{z}-R \omega_{x}\right)\left(\delta u_{z}-R \delta \omega_{x}\right) d x=0
\end{aligned}
$$

where the compressive force $P=\Lambda A$ will act further as the bifurcation parameter.

First, by integrating over the cross-section and eliminating negligible higher-order terms (presupposing that $\left.\Lambda_{c} \ll E, G\right)$, the bifurcation equation 15 can be simplified into:

$$
\begin{gathered}
\forall \delta u_{x}, \delta u_{z}, \delta \omega_{x} \quad(\mathrm{KA}), \quad \int_{0}^{L}\left[E A u_{x},,_{x} \delta u_{x},_{x}+E I u_{z, x x} \delta u_{z, x x}+G J \omega_{x},_{x} \delta \omega_{x},_{x}\right. \\
\left.-P_{c} u_{z,{ }_{x}} \delta u_{z, x}+k\left(u_{z}-R \omega_{x}\right)\left(\delta u_{z}-R \delta \omega_{x}\right)\right] d x=0
\end{gathered}
$$


Then, integrating by parts with respect to $x$ yields three local differential equations for the components $u_{x}, u_{z}$ and $\omega_{x}$ of the eigenmode:

$$
\begin{aligned}
& E A u_{x},_{x x}=0 \\
& E I u_{z, x x x x}+P_{c} u_{z, x x}+k\left(u_{z}-R \omega_{x}\right)=0 \\
& G J \omega_{x}, x x+k R\left(u_{z}-R \omega_{x}\right)=0
\end{aligned}
$$

together with the natural boundary conditions, which will be specified later when necessary.

\subsection{Solution procedure}

The first differential equation in 17 is decoupled from the two others and it leads to the trivial solution $u_{x}=0$ whatever the boundary conditions. The last two equations are coupled with each other, since they both involve the lateral displacement field $u_{z}$ and the rotation $\omega_{x}$. The sought buckling modes are thus prone to combine bending and torsion.

At this stage, in order to facilitate further analysis, a set of dimensionless parameters is defined:

$$
\begin{aligned}
& \alpha=\frac{I}{A R^{2}} \\
& \beta=\frac{G}{E} \\
& \gamma=\frac{A L^{2}}{I} \\
& \xi=\frac{k R^{2}}{E A} \\
& \bar{P}=\frac{P}{E A}
\end{aligned}
$$

and the last two differential equations in (17) can be re-written as follows:

$$
\begin{aligned}
& \frac{1}{\gamma} \bar{u}_{z, \bar{x} \bar{x} \bar{x} \bar{x}}+\bar{P}_{c} \bar{u}_{z, \bar{x} \bar{x}}+\sqrt{\alpha \gamma} \xi\left(\sqrt{\alpha \gamma} \bar{u}_{z}-\omega_{x}\right)=0 \\
& 2 \beta \omega_{x, \bar{x} \bar{x}}+\gamma \xi\left(\sqrt{\alpha \gamma} \bar{u}_{z}-\omega_{x}\right)=0
\end{aligned}
$$

where $\bar{x}=\frac{x}{L}$ is the new dimensionless longitudinal coordinate and the modal displacement $u_{z}$ has been replaced by the dimensionless field $\bar{u}_{z}=\frac{u_{z}}{L}$.

The equation system 19 will be solved below for two particular choices of boundary conditions: concerning the tranverse displacement, simply-supported conditions will be equally assumed in both cases (as already considered in many previous studies, such as [39, 40]), whereas the torsional rotation at the ends of the beam will be either fixed or free. All the subsequent results are applicable, provided that the following condition is fulfilled (which is always the case in practice):

$$
\xi<4 \alpha \beta^{2}
$$

\subsubsection{Simply-supported conditions without torsional rotation}

In the present case, the components of the eigenmode must satisfy the following displacement boundary conditions at both ends $\bar{x}=0$ and $\bar{x}=1: \bar{u}_{z}(0)=0, \bar{u}_{z}(1)=0, \omega_{x}(0)=0$ and $\omega_{x}(1)=0$. The remaining 
stress boundary conditions stemming from the integration by parts of the bifurcation equation are written thus: $\bar{u}_{z, \bar{x} \bar{x}}(0)=0$ and $\bar{u}_{z, \bar{x} \bar{x}}(1)=0$.

It can be shown that, with the boundary conditions defined above, the modal transverse displacement takes necessarily the same sinusoidal form as the buckling mode of a single beam under axial compression, that is to say:

$$
\bar{u}_{z}=\sin (n \pi \bar{x})
$$

where $n$ represents the half-wave number of the mode. The torsional component of the mode is also found to be sinusoidal (which is naturally consistent with the fixed rotations at the ends) and a closed-form solution can be obtained for the corresponding dimensionless critical force (or critical strain):

$$
\bar{P}_{c}(n)=\frac{n^{2} \pi^{2}}{\gamma}+\frac{\alpha \gamma \xi}{n^{2} \pi^{2}+\frac{\gamma \xi}{2 \beta}}
$$

which also depends on the half-wave number $n$ of the buckling mode.

From Equation (22), the minimum critical value (corresponding to the first buckling mode) is obtained by minimizing $\bar{P}_{c}(n)$ with respect to the integer $n$.

\subsubsection{Simply-supported conditions with free torsional rotation}

Let us now consider the case with similar simply-supported conditions, as far as bending is concerned, but free torsional rotation at both ends. In this context, two different mode types are encountered, that are decoupled from each other. The buckling modes are alternately symmetric and antisymmetric with respect to the middle of the beam: the antisymmetric modes display a classical sinusoidal shape as obtained before (with the same critical load), whereas the previous sinusoidal symmetric modes are replaced here by more complex solutions involving hyperbolic functions. The existence of such a symmetric mode is guaranteed once the following conditions are fulfilled:

$$
\begin{aligned}
& \xi \geq \xi_{\text {min }}=\alpha \beta^{2}\left[1-\sqrt{1-\left(\frac{\eta}{\chi}\right)^{2}}\right]^{2} \\
& \chi \geq \frac{\pi}{2}
\end{aligned}
$$

where $\chi=\sqrt{\frac{\alpha \beta \gamma}{8}}$ and $\eta$ is the real positive solution of the transcendental equation:

$$
\eta-\sin (2 \eta)\left[1+\frac{1}{2} \sqrt{1-\left(\frac{\eta}{\chi}\right)^{2}}\right]=0
$$

The first inequality in 23 will be verified in the practical applications, when considering usual values for the stiffness of lateral connectors, whereas the second inequality is mainly related to the slenderness of the pipe and is naturally satisfied for current geometries.

As the first mode is symmetric in practice (combined with the fact that the antisymmetric modes and corresponding critical loads have been fully described above), one will only be interested here in symmetric 
modes. Considering now, for simplicity purposes, that the middle of the beam corresponds to the longitudinal coordinate $\bar{x}=0$, only even functions will be involved in the sought buckling modes. Hence, the general symmetric solution of the equation system (19) for the lateral displacement turns out to be in the following form:

$$
\bar{u}_{z}=c_{0}+c_{1} \cosh (a \bar{x}) \cos (b \bar{x})+c_{2} \sinh (a \bar{x}) \sin (b \bar{x})
$$

where the coefficients $c_{0}, c_{1}$ and $c_{2}$ are constants to be defined and the arguments $a$ and $b$, respectively in the hyperbolic and sinusoidal functions, can be expressed in terms of the dimensionless parameters as follows:

$$
\begin{aligned}
& a=\frac{1}{2 \sqrt{2}} \sqrt{\frac{\gamma}{\beta}} \sqrt{2 \sqrt{2 \beta \xi\left(2 \alpha \beta-\bar{P}_{c}\right)}+\xi-2 \beta \bar{P}_{c}} \\
& b=\frac{1}{2 \sqrt{2}} \sqrt{\frac{\gamma}{\beta}} \sqrt{2 \sqrt{2 \beta \xi\left(2 \alpha \beta-\bar{P}_{c}\right)}+2 \beta \bar{P}_{c}-\xi}
\end{aligned}
$$

A similar expression (with other coefficients) is obtained for the torsional rotation.

In this new case, the components of the eigenmode must satisfy the following displacement boundary conditions at both ends $\bar{x}=-\frac{1}{2}$ and $\bar{x}=\frac{1}{2}: \bar{u}_{z}\left(-\frac{1}{2}\right)=0, \bar{u}_{z}\left(\frac{1}{2}\right)=0$. The remaining stress boundary conditions stemming from the integration by parts of the bifurcation equation write: $\bar{u}_{z, \bar{x} \bar{x}}\left(-\frac{1}{2}\right)=0$, $\bar{u}_{z, \bar{x} \bar{x}}\left(\frac{1}{2}\right)=0, \omega_{x}, \bar{x}\left(-\frac{1}{2}\right)=0$ and $\omega_{x}, \bar{x}\left(\frac{1}{2}\right)=0$. Since both fields $\bar{u}_{z}$ and $\omega_{x}$, as well as their derivatives, are either even or odd functions with respect to $\bar{x}$, the boundary conditions can only be written at one end of the beam, say at $\bar{x}=\frac{1}{2}$.

These three boundary conditions form a linear equation system depending on the three constants $c_{0}, c_{1}$ and $c_{2}$. One obtains the critical strain $\bar{P}_{c}$ by setting the determinant of this linear equation system to zero, namely by solving the following characteristic equation:

$$
\begin{gathered}
b\left[-\alpha \gamma^{2} \xi+\bar{P}_{c} \gamma\left(a^{2}+b^{2}\right)+\left(a^{2}+b^{2}\right)\left(3 a^{2}-b^{2}\right)\right] \sinh (a) \\
+a\left[\alpha \gamma^{2} \xi+\bar{P}_{c} \gamma\left(a^{2}+b^{2}\right)+\left(a^{2}+b^{2}\right)\left(a^{2}-3 b^{2}\right)\right] \sin (b)=0
\end{gathered}
$$

\subsection{Connection with the neutral axis}

In the case where the connection to the ground is applied on the neutral axis of the beam, the kinematics is the same as before (see Equation (11) ) but the transverse slip $g$ is reduced here to the following expression:

$$
g=u_{z}
$$


The system of differential equations 17 is thus replaced by:

$$
\begin{aligned}
& E A u_{x}, x_{x}=0 \\
& E I u_{z, x x x x}+P_{c} u_{z}, x x+k u_{z}=0 \\
& G J \omega_{x},,_{x x}=0
\end{aligned}
$$

The first and third differential equations in 30 are decoupled from the others and lead to the trivial solution $u_{x}=0$ and $\omega_{x}=0$. The last equation only involves $u_{z}$, so that only flexural buckling is expected (without torsional effects). Using the same dimensionless parameters as before, this unique equation writes:

$$
\frac{1}{\gamma} \bar{u}_{z, \bar{x} \bar{x} \bar{x} \bar{x}}+\bar{P}_{c} \bar{u}_{z, \bar{x} \bar{x}}+\alpha \gamma \xi \bar{u}_{z}=0
$$

When solving Equation (31), one has necessarily to set the torsional rotation to zero at both ends in order to satisfy the trivial solution $\omega_{x}=0$, namely to prevent from a rotation rigid mode. Furthermore, simply-supported conditions are still considered and the same boundary conditions are used as in Subsection 2.3.1. $\bar{u}_{z}(0)=0, \bar{u}_{z}(1)=0, \bar{u}_{z, \bar{x} \bar{x}}(0)=0$ and $\bar{u}_{z, \bar{x} \bar{x}}(1)=0$.

The modal transverse displacement takes the same sinusoidal form as before:

$$
\bar{u}_{z}=\sin (n \pi \bar{x})
$$

and a closed-form solution is obtained again for the critical strain:

$$
\bar{P}_{c}(n)=\frac{n^{2} \pi^{2}}{\gamma}+\frac{\alpha \gamma \xi}{n^{2} \pi^{2}}
$$

which is identical to the solution initially found by Timoshenko and Gere 41], among others.

When comparing expressions $(22)$ and (33), one observes that the critical loads obtained in the case of a connection with the bottom line of the beam (but without torsional rotation at the ends) are necessarily lower than the ones obtained in the case of a connection with the neutral axis. However, owing to the very low values of the ratio $\frac{\gamma \xi}{2 \beta}$ encountered in practice, the critical loads will almost coincide between the two cases.

\subsection{Special case with a vanishing connection stiffness density}

Before a more general numerical validation of the previous analytical solutions, it could be interesting to consider the special case of a null stiffness density $(k=0)$ for which the tube is supposed to behave simply like a beam under axial compression. Considering that $\xi=0$ in Equations $(22)$ and $(33)$ leads to the same simplified expression of the critical strain:

$$
\bar{P}_{c}(n)=\frac{n^{2} \pi^{2}}{\gamma}
$$

This solution is consistent with the well-known critical force of an Euler-Bernoulli simply-supported beam under axial compression:

$$
P_{c}(n)=\frac{n^{2} \pi^{2} E I}{L^{2}}
$$

which is naturally minimum for $n=1$. 


\section{Numerical validation and analysis}

\subsection{Numerical modeling}

In order to reinforce the analytical solutions obtained above for the critical values and buckling modes under various boundary conditions, finite element computations have been performed using Abaqus software. Shear-flexible linear (two-node) beam elements in 3D space (B31 in Abaqus) have been retained since EulerBernoulli beam elements (with cubic Hermite interpolation functions) may not be appropriate for torsional stability problems due to the approximations in the underlying formulation. The geometric and material parameters are summarized in Table 1. The whole model and especially the boundary conditions are represented in Figure 2, Vertical displacements (in $y$-direction) have been set to zero in the whole beam, since upheaval of the tube is not permitted. A null lateral displacement (in $z$-direction) has been enforced at both ends so as to conform to the simply-supported boundary conditions and the left end has been fixed longitudinally in order to avoid any rigid mode. Last, both ends have been prevented (or not) from rotation about the longitudinal axis of the beam, depending on the considered case.

\begin{tabular}{|c|c|c|c|c|}
\hline Length & Internal radius & External radius & Young's modulus & Poisson's ratio \\
\hline $100 \mathrm{~m}$ & $145 \mathrm{~mm}$ & $162 \mathrm{~mm}$ & $210000 \mathrm{MPa}$ & 0.3 \\
\hline
\end{tabular}

Table 1: Geometric and material parameters for the numerical validation

The beam is then related to the ground by specific connector elements. Discrete connectors are applied at each node of the beam so as to reproduce the theoretically continuous distribution of springs. In practice, the discrete stiffness $K_{d}$ at each node (in $N / m$ ) equals the stiffness density by unit length $k$ (in $N / m^{2}$ ) multiplied by the length of each element (between connectors). The beam model is classically represented by its neutral axis, so that it is more convenient to rely the connectors to this line. Thus, a coupled set of return springs has been used so as to simulate a single lateral spring connected to the bottom line of the beam. The linear connection is actually defined through the following stiffness matrix relating the lateral force and torsional moment exerted by the ground to the lateral displacement and torsional rotation as follows:

$$
\left\{\begin{array}{c}
F_{z} \\
M_{x}
\end{array}\right\}=\left[\begin{array}{cc}
K_{d} & -K_{d} R \\
-K_{d} R & K_{d} R^{2}
\end{array}\right]\left\{\begin{array}{c}
u_{z} \\
\omega_{x}
\end{array}\right\}
$$

In the particular case where the connection is really applied to the neutral axis, Equation $(36)$ must be replaced by the single relation:

$$
F_{z}=K_{d} u_{z}
$$

Let us recall that the fundamental criterion that allows one to neglect transverse shear effects in the 


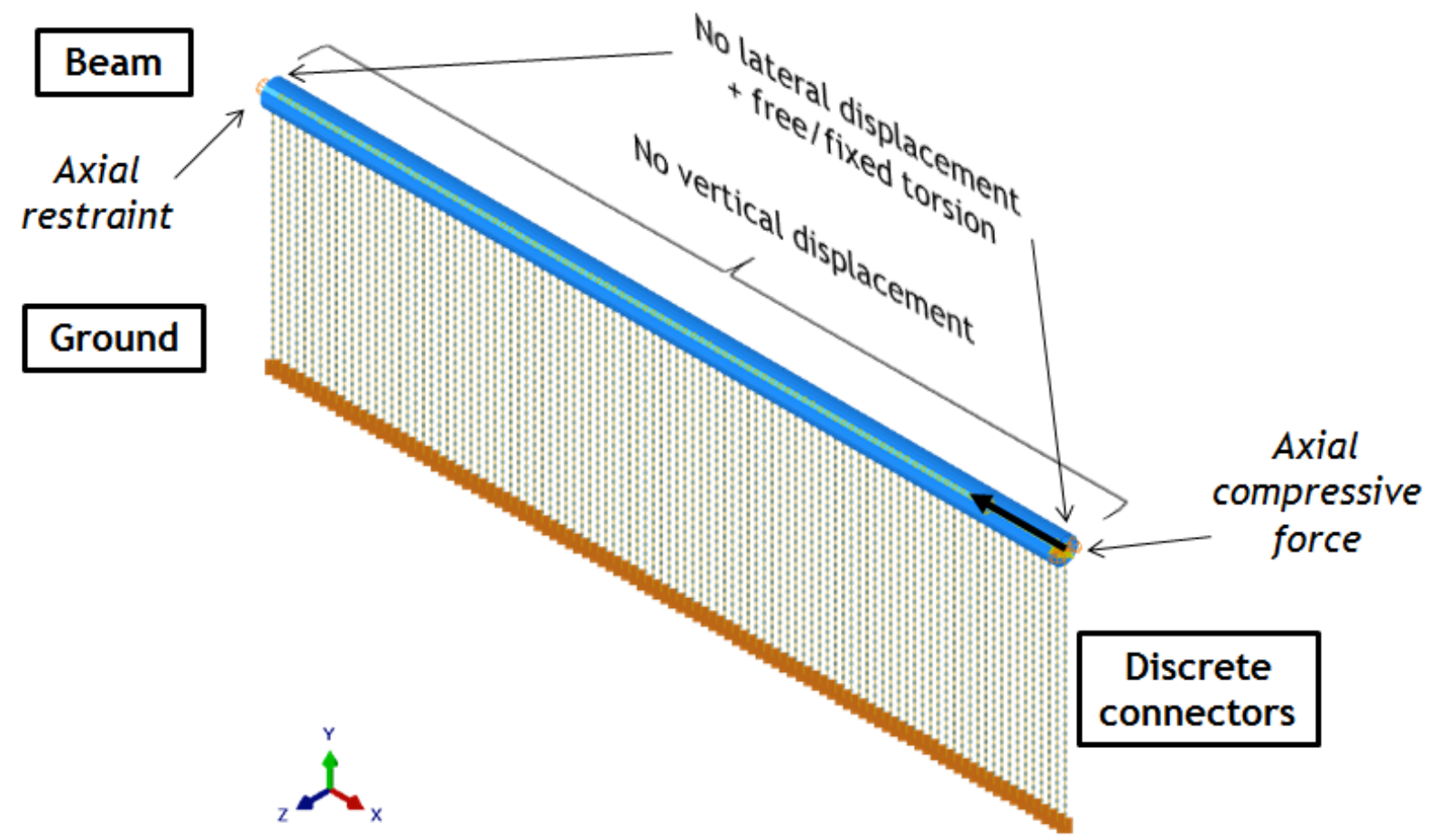

Figure 2: Finite element model and boundary conditions

bending response of a beam can be expressed as follows:

$$
\frac{12}{\beta \gamma}=\frac{12 E I}{G A L^{2}} \ll 1
$$

With the geometric and material parameters considered in this study (see Table 1), this ratio takes the particular value of $3.710^{-5}$, what confirms the appropriate use of an Euler-Bernoulli beam model.

Furthermore, in the following investigations, the dimensionless parameter $\xi$ standing for the lateral stiffness density varies from $10^{-9}$ to $10^{-7}$ which corresponds to realistic values, given the pipe-soil friction coefficient encountered in practice. The current geometric and material parameters lead to the following values:

$$
4 \alpha \beta^{2}=0.266 \quad \chi=135 \quad \xi_{\text {min }}=8.3610^{-11}
$$

so that conditions $(20)$ and $(23)$ are largely satisfied.

A reference (unit) compressive force is finally applied to the right end of the beam and a linearized stability analysis is achieved, where the critical load factors are hence found to be the sought critical loads.

\subsection{Validation of the analytical solutions}

First of all, numerical computations are performed in the three previous configurations which gave rise to analytical solutions: two of them correspond to the new model with connection to the bottom line of the 
pipe, with either fixed or free torsional rotation at the ends, and the third one corresponds to the classical model with connection to the neutral axis. In each case, five particular values of stiffness density have been considered, corresponding to $\xi=10^{-9}, 10^{-8}, 4.10^{-8}, 7.10^{-8}, 10^{-7}$.

A short mesh sensitivity analysis has been preliminary achieved so as to get converged results and also verify the ability to reproduce the continuous distribution of springs with discrete connectors (one per node). For illustration purposes, Table 2 displays the critical forces, in the case of free torsional rotation with $\xi=10^{-7}$, obtained respectively for 25,50 and 100 elements. A fine mesh of 100 elements of equal length (namely $1 \mathrm{~m}$ ) has been finally retained, in such a way that the discrete stiffness $K_{d}$ takes the same value as the stiffness density by unit length $k$.

\begin{tabular}{|c|c|c|c|}
\hline Number of elements & 25 & 50 & 100 \\
\hline Critical force (N) & 770516 & 739349 & 731581 \\
\hline
\end{tabular}

Table 2: Mesh convergence study

All the analytical and numerical results corresponding to these 15 examples have been put together in Table 3, namely the minimum critical forces $P_{c}$ but also the associated dimensionless critical compressive strains $\bar{P}_{c}$, together with the relative errors between analytical and numerical predictions. In the cases where the mode is sinusoidal and depends on a wave number $n$, the value $n_{\text {opt }}$ which minimizes the buckling load has also been indicated. In all cases, analytical and numerical results are shown to be in very good agreement, with a maximum relative error of $0.5 \%$.

Figure 3 depicts, for the three configurations investigated, the evolution of the analytical minimum critical strain (corresponding to the first buckling mode) with respect to parameter $\xi$. In each case, the five numerical values have been plotted on the same graphs for completeness purposes. In Figure 3(c), the curve is piecewise linear, as expected by Equation (33). The same tendency is observed in Figure 3 (a), even if Equation $(22)$ does not display a linear form. In both cases, the slope changes correspond to the incrementation by one unit of the optimal wave number $n_{\text {opt }}$. Finally, the analytical and numerical buckling mode shapes have been compared to each other, still for the three configurations considered (with $\xi=10^{-7}$ ). Figure 4 also shows a very good accordance in all cases. One can mention here that the present modes, whatever the boundary conditions, slightly differ from classical modes observed in 23, among others. It is due to the fact that axial friction effects have not been taken into account in the present analysis, instead a finite pipe has been considered with specific boundary conditions applied at its ends. These particular mode shapes will be employed alongside with the other classical localized modes encountered in the literature so as to investigate the significant influence of geometric imperfections on the lateral buckling of such pipes in further studies. 


\begin{tabular}{|c|c|c|c|c|c|c|c|}
\hline$\xi$ & $\begin{array}{c}\text { Stiffness density } \\
\quad k\left(\text { in } N / m^{2}\right)\end{array}$ & $\begin{array}{l}\text { FE critical } \\
\text { force }(\text { in } N)\end{array}$ & $\begin{array}{l}\text { FE critical } \\
\text { strain } \bar{P}_{c}^{F E}\end{array}$ & $\begin{array}{c}\text { Analytical critical } \\
\text { strain } \bar{P}_{c}\end{array}$ & $\begin{array}{c}\text { Error } \\
\frac{\left\|\bar{P}_{c}-\bar{P}_{c}^{F E}\right\|}{\bar{P}_{F}^{F E}}\end{array}$ & $\begin{array}{l}\text { FE wave } \\
\text { number }\end{array}$ & $\begin{array}{c}\text { Wave } \\
\text { number } n_{\text {opt }}\end{array}$ \\
\hline \multicolumn{8}{|c|}{ Connection to the bottom line with fixed rotation at the ends } \\
\hline $10^{-9}$ & 131.2 & $1.7310^{5}$ & $5.02410^{-5}$ & $5.02710^{-5}$ & $0.051 \%$ & 1 & 1 \\
\hline $10^{-8}$ & 1312 & $4.92510^{5}$ & $1.4310^{-4}$ & $1.43110^{-4}$ & $0.045 \%$ & 2 & 2 \\
\hline $4.10^{-8}$ & 5248 & $9.51310^{5}$ & $2.76310^{-4}$ & $2.76510^{-4}$ & $0.078 \%$ & 3 & 3 \\
\hline $7.10^{-8}$ & 9184 & $1.22310^{6}$ & $3.55110^{-4}$ & $3.55410^{-4}$ & $0.082 \%$ & 4 & 4 \\
\hline $10^{-7}$ & 13120 & $1.47210^{6}$ & $4.27510^{-4}$ & $4.27710^{-4}$ & $0.045 \%$ & 4 & 4 \\
\hline \multicolumn{8}{|c|}{ Connection to the bottom line with free rotation at the ends } \\
\hline $10^{-9}$ & 131.2 & $6.47910^{4}$ & $1.88210^{-5}$ & $1.88410^{-5}$ & $0.12 \%$ & - & - \\
\hline $10^{-8}$ & 1312 & $2.34510^{5}$ & $6.81110^{-5}$ & $6.80310^{-5}$ & $0.12 \%$ & - & - \\
\hline $4.10^{-8}$ & 5248 & $4.62510^{5}$ & $1.34310^{-4}$ & $1.34510^{-4}$ & $0.13 \%$ & - & - \\
\hline $7.10^{-8}$ & 9184 & $6.07610^{5}$ & $1.76510^{-4}$ & $1.76710^{-4}$ & $0.13 \%$ & - & - \\
\hline $10^{-7}$ & 13120 & $7.31610^{5}$ & $2.12510^{-4}$ & $2.11610^{-4}$ & $0.41 \%$ & - & - \\
\hline \multicolumn{8}{|c|}{ Connection to the neutral axis (with fixed rotation at the ends) } \\
\hline $10^{-9}$ & 131.2 & $1.7310^{5}$ & $5.02410^{-5}$ & $5.02710^{-5}$ & $0.051 \%$ & 1 & 1 \\
\hline $10^{-8}$ & 1312 & $4.92610^{5}$ & $1.43110^{-4}$ & $1.43210^{-4}$ & $0.096 \%$ & 2 & 2 \\
\hline $4.10^{-8}$ & 5248 & $9.51610^{5}$ & $2.76410^{-4}$ & $2.76610^{-4}$ & $0.083 \%$ & 3 & 3 \\
\hline $7.10^{-8}$ & 9184 & $1.22310^{6}$ & $3.55210^{-4}$ & $3.55510^{-4}$ & $0.087 \%$ & 4 & 4 \\
\hline $10^{-7}$ & 13120 & $1.47310^{6}$ & $4.27710^{-4}$ & $4.27910^{-4}$ & $0.052 \%$ & 4 & 4 \\
\hline
\end{tabular}

Table 3: Analytical and numerical minimum critical values

\subsection{Comparison between the two modeling approaches}

It is interesting now to compare the previous analytical solutions with each other. First, the two types of connection (on the bottom line and on the neutral axis) are considered, with the same boundary conditions (namely fixed transverse displacement and fixed torsional rotation at both ends). Figure 5 displays the evolution of the corresponding critical strains with parameter $\xi$ and shows that the two curves are nearly coincident between the two types of connection. In Table 3, the critical loads had already been found identical between the two models (for the values of $\xi$ considered) together with the wave numbers which were also shown to be equal. It means that, in the range of parameters considered and for these particular boundary conditions, torsion has no particular influence on the critical load of the beam. In Equation 22 . formulating the critical load of the beam with torsional effects, due to the very low value of the ratio $\frac{\gamma \xi}{2 \beta \pi^{2}}$ (which equals 0.0111 for the maximum stiffness considered, namely $\xi=10^{-7}$ ), one can neglect the second 

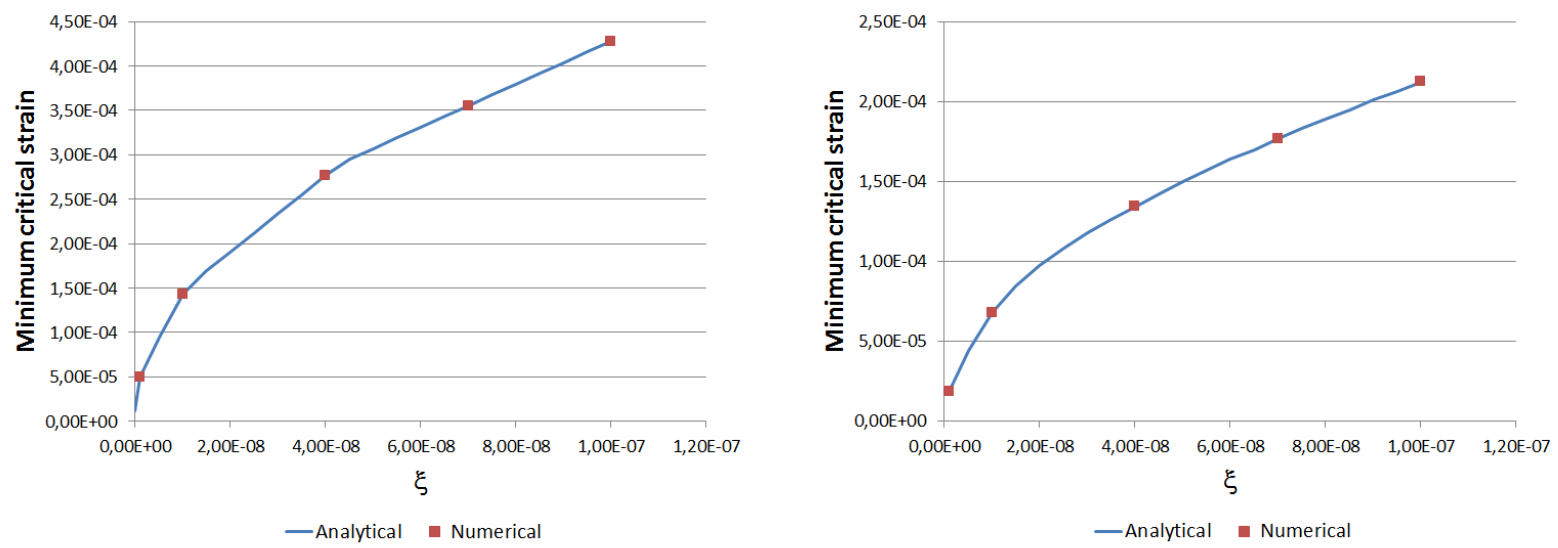

(a) Connection to the bottom line with fixed rotation at the (b) Connection to the bottom line with free rotation at the ends ends

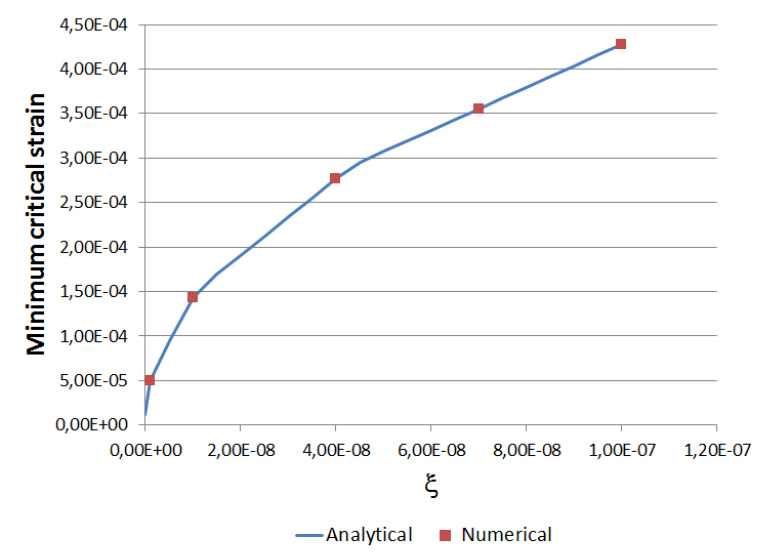

(c) Connection to the neutral axis (with fixed rotation at the ends)

Figure 3: Analytical and numerical minimum critical strains vs. stiffness parameter $\xi$

term of the denominator $\left(\frac{\gamma \xi}{2 \beta}\right)$ with respect to the first one, what leads precisely to the expression of Equation (33) excluding the torsional effects.

In Figure 5, the critical loads obtained with the other boundary conditions (namely free torsional rotation at the ends) are also plotted, for comparison purposes. When the beam is connected to the ground with its neutral axis, a rigid mode of axial rotation of the beam may appear when letting free the rotation in question at both ends. The critical loads and corresponding buckling modes are thus unchanged, as soon as one sets the axial rotation to zero at one end at least, to prevent from the rigid mode. Conversely, when the beam is connected to the ground with its bottom line, there is no rigid mode even when the torsional rotation is free at both ends. In these conditions, as seen in Figure 5, the critical loads are about half than the previous 

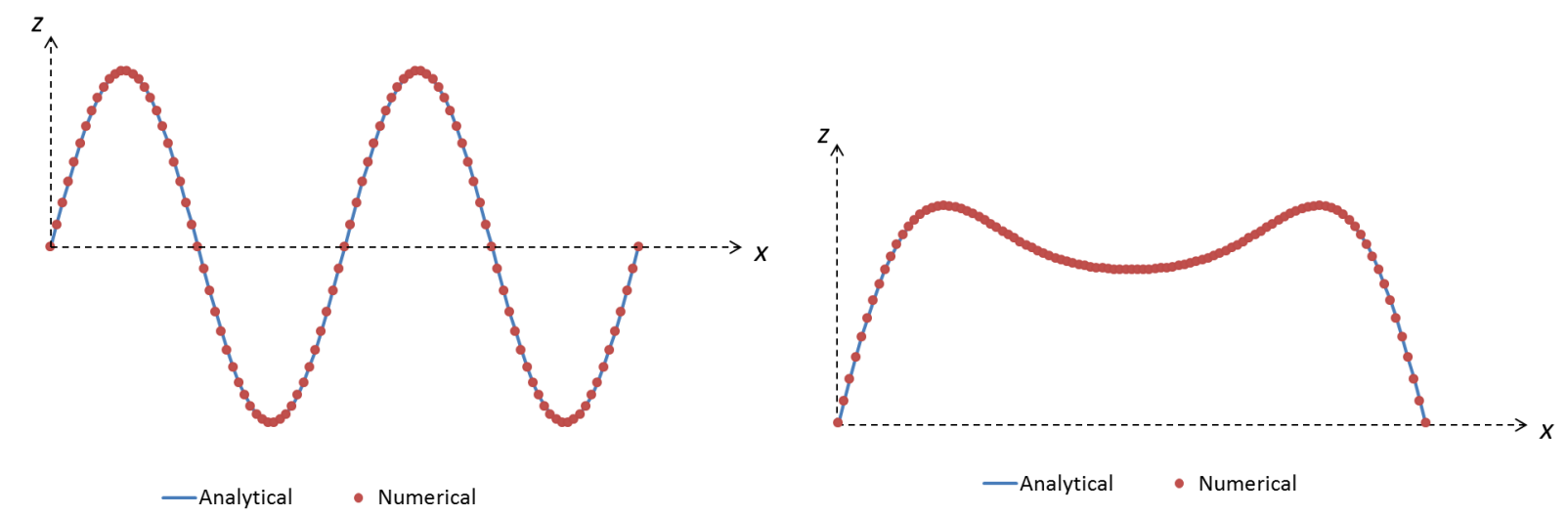

(a) Connection to the bottom line with fixed rotation at the (b) Connection to the bottom line with free rotation at the ends ends

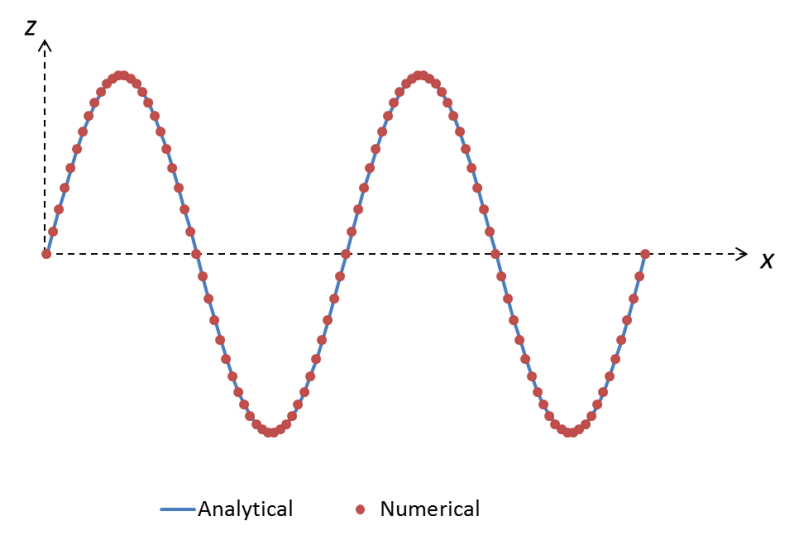

(c) Connection to the neutral axis (with fixed rotation at the ends)

Figure 4: Analytical and numerical first buckling modes for $\xi=10^{-7}$

values, other things being equal. Therefore, in such conditions, it turns out to be very important to take into account torsional effects in the lateral global buckling analysis of the tube.

\section{Conclusion}

In this paper, a new simple problem has been investigated as a first step towards the analysis of the lateral buckling of submarine pipelines resting on the seabed. The pipe is here represented by an elastic Euler-Bernoulli beam under axial compression (which is supposed to derive from both internal/external pressure and thermal expansion effects in practice), and the pipe-soil interaction, which strongly influences the buckling response, is modeled through a continuous spring distribution also displaying an elastic consti- 


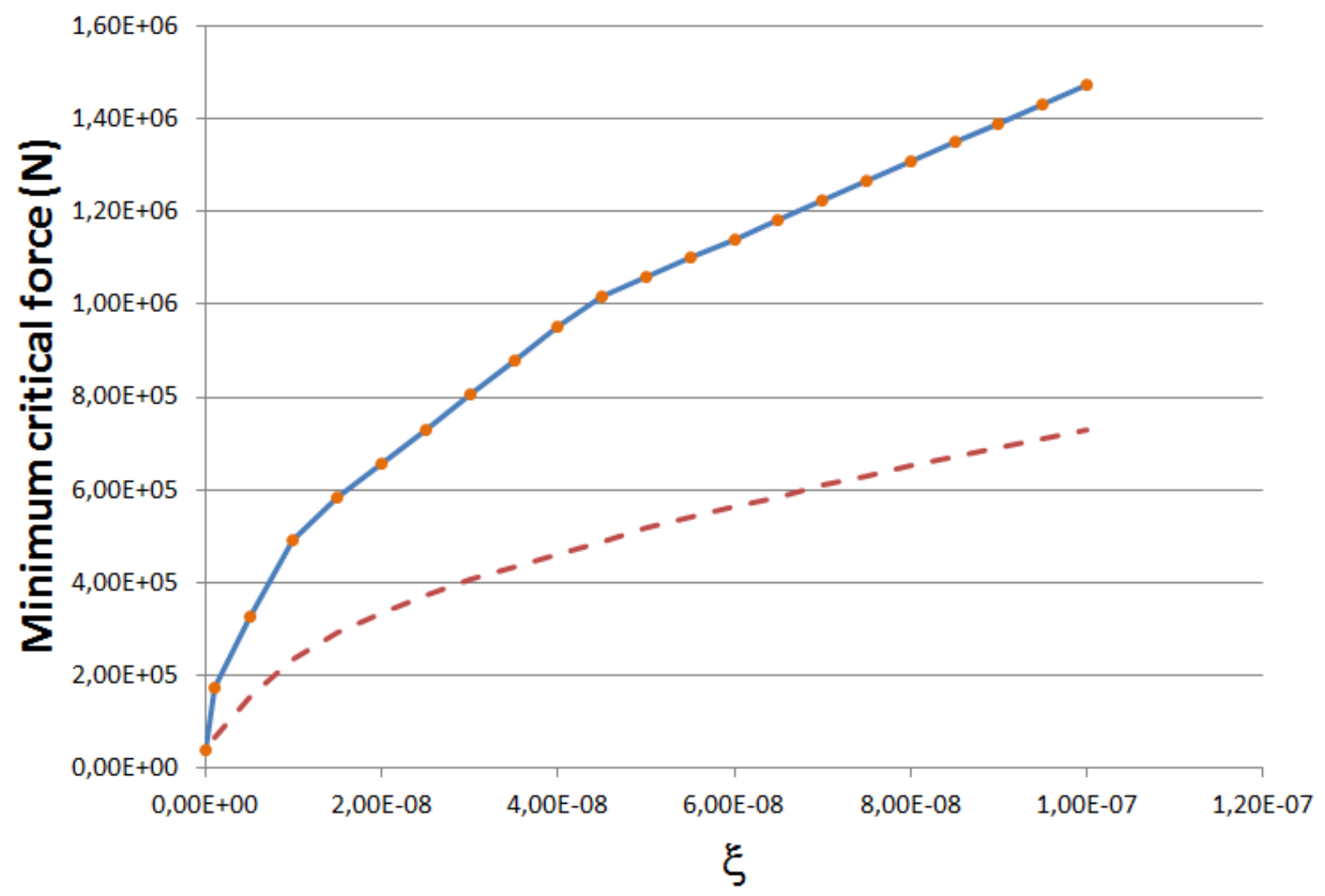

— Bottom line-fixed rotation - - Bottom line-free rotation - Neutral axis

Figure 5: Comparisons between the different analytical critical forces

tutive law. The present model is original in that the connection is achieved between the supposedly rigid soil and the bottom line of the tube in real contact with the seabed (and not the neutral axis of the beam as it is currently the case in the literature), allowing thus for torsional effects. The buckling problem is solved analytically, using the so-called bifurcation equation, what leads to the critical forces and corresponding modes.

These analytical results are reinforced by comparison with numerical finite element computations performed using a commercial software (Abaqus). Two sets of boundary conditions are therefore considered, namely simply-supported ends with or without torsional restraints at the ends. While, in the former case, the buckling modes display a sinusoidal deformed shape and the critical forces hardly differ from the ones classically obtained when torsional effects are neglected, in the latter case, when the ends are free in torsion, the buckling modes (and specifically the first one) take a different shape and, what is more, the associated critical loads are found to be halved, roughly speaking.

This first simple model highlights thus the essential role of torsion in the lateral buckling phenomenon, 
at least under particular boundary conditions. In practice, a more realistic analysis of the lateral buckling of submarine pipelines requires the use of a more comprehensive model, involving new features such as (axial) friction effects [42, material and geometric non-linearities, among other things, but there is no doubt that torsion will also influence the global/localized buckling response of such practical pipelines. Further analyses are currently performed in that direction so as to get closer to real conditions, in terms of constitutive laws, geometric imperfections, boundary conditions and initial conditions (such as residual stresses), still considering the crucial influence of torsional effects, which should result in a new paper.

\section{References}

[1] Bai, Y., Igland, R. and Moan, T., Tube collapse under pressure, tension and bending loads, International Journal of Offshore and Polar Engineering 3 Issue 2 (1993) 121-129.

[2] Taylor, N. and Gan, A.B., Submarine pipeline buckling - imperfection studies, Thin-Walled Structures 4 Issue 4 (1986) 295-323.

[3] Karampour, H., Albermani, F. and Gross, J., On lateral and upheaval buckling of subsea pipelines, Engineering Structures 52 (2013) 317-330.

[4] Hunt, G.W. and Blackmore, A., Principles of localized buckling for a strut on an elastoplastic foundation, Journal of Applied Mechanics 63 Issue 1 (1996) 234-239.

[5] Wang, Z. and van der Heijden, G.H.M., Localised lateral buckling of partially embedded subsea pipelines with nonlinear soil resistance, Thin-Walled Structures 120 (2017) 408-420.

[6] Cai, J., Jiang, X. and Lodewijks, G., Residual ultimate strength of offshore metallic pipelines with structural damage - a literature review, Ships and Offshore Structures 12 Issue 8 (2017) 1037-1055.

[7] Cai, J., Jiang, X., Lodewijks, G., Pei, Z. and Wu, W., Residual ultimate strength of damaged seamless metallic pipelines with combined dent and metal loss, Marine Structures 61 (2018) 188-201.

[8] Palmer, A.C. and Baldry, J.A.S., Lateral buckling of axially constrained pipelines, Journal of Petroleum Technology 26 Issue 11 (1974) 1283-1284.

[9] Fyrileiv, O. and Collberg, L., Influence of pressure in pipeline design: Effective axial force, ASME 24 ${ }^{\text {th }}$ International Conference on Offshore Mechanics and Arctic Engineering Halkidiki, Greece, June 12-17, 2005.

[10] Vedeld, K., Sollund, H.A., Hellesland, J. and Fyrileiv, O., Effective axial forces in offshore lined and clad pipes, Engineering Structures 66 (2014) 66-80.

[11] Galgoul, N.S., Massa, A.L.L. and Claro, C.A., Lateral buckling: Trying to be less conservative, ASME International Pipeline Conference Calgary, Alberta (Canada), October 4-8, 2004

[12] Murff, J.D., Wagner, D.A. and Randolph, M.F., Pipe penetration in cohesive soil, Géotechnique 39 Issue 2 (1989) $213-229$.

[13] Bruton, D., White, D.J., Cheuk, C.Y., Bolton, M.D. and Carr, M., Pipe/soil interaction behavior during lateral buckling, including large-amplitude cyclic displacement tests by the safebuck JIP, Offshore Technology Conference Houston, Texas (USA), May 1-4, 2006.

[14] Schotman, G.J.M. and Stork, F.G., Pipe-soil interaction: A model for laterally loaded pipelines in clay, Offshore Technology Conference Houston, Texas (USA), April 27-30, 1987.

[15] Zhang, J., Stewart, D.P. and Randolph, M.F., Kinematic hardening model for pipeline-soil interaction under various loading conditions, International Journal of Geomechanics 2 Issue 4 (2002) 419-446.

[16] White, D.J. and Cheuk, C.Y., Modelling the soil resistance on seabed pipelines during large cycles of lateral movement, Marine Structures 21 Issue 1 (2008) 59-79. 
[17] Cheuk, C.Y. and Bolton, M.D., A technique for modelling the lateral stability of on-bottom pipelines in a small drum centrifuge, $6^{\text {th }}$ International Conference on Physical Modelling in Geotechnics Hong-Kong, August 4-6, 2006.

[18] Cheuk, C.Y., White, D.J. and Bolton, M.D., Large-scale modelling of soil-pipe interaction during large amplitude cyclic movements of partially embedded pipelines, Canadian Geotechnical Journal 44 Issue 8 (2007) 977-996.

[19] Zhang, Z., Liu, H. and Chen, Z., Lateral buckling theory and experimental study on pipe-in-pipe structure, Metals 9 Issue 2 (2019) 185-200.

[20] Wang, L., Shi, R., Yuan, F., Guo, Z. and Yu, L., Global buckling of pipelines in the vertical plane with a soft seabed, Applied Ocean Research 33 Issue 2 (2011) 130-136.

[21] Zeng, X. and Duan, M., Mode localization in lateral buckling of partially embedded submarine pipelines, International Journal of Solids and Structures 51 Issue 10 (2014) 1991-1999.

[22] Hobbs, R.E., Pipeline buckling caused by axial loads, Journal of Constructional Steel Research 1 Issue 2 (1981) 2-10.

[23] Hobbs, R.E., In-service buckling of heated pipelines, Journal of Transportation Engineering 110 Issue 2 (1984) $175-189$.

[24] Ju, G.T. and Kyriakides, S., Thermal buckling of offshore pipelines, Journal of Offshore Mechanics and Arctic Engineering 110 Issue 4 (1988) 355-364.

[25] Taylor, N. and Tran, V., Prop-imperfection subsea pipeline buckling, Marine Structures 6 Issue 4 (1993) $325-358$.

[26] Hong, Z., Liu, R., Liu, W. and Yan, S., Study on lateral buckling characteristics of a submarine pipeline with a single arch symmetric initial imperfection, Ocean Engineering 108 (2015) 21-32.

[27] Kerr, A.D., Analysis of thermal track buckling in the lateral plane, Acta Mechanica 30 Issues 1-2 (1978) 17-50.

[28] Miles, D.J. and Calladine, C.R., Lateral thermal buckling of pipelines on the sea bed, Journal of Applied Mechanics 66 Issue 4 (1999) 891-897.

[29] Zhang, X. and Duan, M., Prediction of the upheaval buckling critical force for imperfect submarine pipelines, Ocean Engineering 109 (2015) 330-343.

[30] Zhu, J., Attard, M.M. and Kellermann, D.C., In-plane nonlinear localised lateral buckling of straight pipelines, Engineering Structures 103 (2015) 37-52.

[31] Liu, R. and Wang, X., Lateral global buckling high-order mode analysis of a submarine pipeline with imperfection, Applied Ocean Research $\mathbf{7 3}$ (2018) 107-126.

[32] Zhang, X., Soares, C.G., An, C. and Duan, M., An unified formula for the critical force of lateral buckling of imperfect submarine pipelines, Ocean Engineering 166 (2018) 324-335.

[33] Le Grognec, P. and Le van, A., On the plastic bifurcation and post-bifurcation of axially compressed beams, International Journal of Non-Linear Mechanics 46 Issue 5 (2011) 693-702.

[34] Douville, M.A. and Le Grognec, P., Exact analytical solutions for the local and global buckling of sandwich beam-columns under various loadings, International Journal of Solids and Structures 50 Issues 16-17 (2013) 2597-2609.

[35] Le Grognec, P. and Sad Saoud, K., Elastoplastic buckling and post-buckling analysis of sandwich columns, International Journal of Non-Linear Mechanics 72 (2015) 67-79.

[36] Le Grognec, P., Nguyen, Q.H. and Hjiaj, M., Exact buckling solution for two-layer Timoshenko beams with interlayer slip, International Journal of Solids and Structures 49 Issue 1 (2012) 143-150.

[37] Le Grognec, P., Nguyen, Q.H. and Hjiaj, M., Plastic bifurcation analysis of a two-layer shear-deformable beam-column with partial interaction, International Journal of Non-Linear Mechanics 67 (2014) 85-94.

[38] Nguyen, Q.S., Stability and Non-Linear Solid Mechanics (Wiley, 2000).

[39] Karampour, H., Albermani, F. and Major, P., Interaction between lateral buckling and propagation buckling in textured deep subsea pipelines, ASME $34^{\text {th }}$ International Conference on Ocean, Offshore and Arctic Engineering St. John's, Newfoundland, Canada, May 31-June 5, 2015.

[40] Karampour, H., Effect of proximity of imperfections on buckle interaction in deep subsea pipelines, Marine Structures $\mathbf{5 9}$ 
(2018) 444-457.

[41] Timoshenko, S.P. and Gere, J.M., Theory of Elastic Stability (Mc Graw-Hill, 1961).

[42] Wang, Z., van der Heijden, G.H.M. and Tang, Y., Localised upheaval buckling of buried subsea pipelines, Marine Structures 60 (2018) 165-185 